\title{
Prevalence and Genotyping of Pneumocystis jirovecii Pneumonia in Patients with Previously Untreated Acute Myeloid Leukemia ${ }^{\dagger}$
}

\author{
Valentina Del Prete ${ }^{1}$, Giovangiacinto Paterno ${ }^{2, *}$, David Di Cave ${ }^{1}$, Luca Guarnera ${ }^{2}$, Raffaele Palmieri ${ }^{2}(\mathbb{D}$, \\ Luca Maurillo ${ }^{2}$, Francesco Buccisano ${ }^{2}\left[\right.$, Adriano Venditti ${ }^{2} \mathbb{D}$ and Maria Ilaria Del Principe ${ }^{2}$ \\ 1 Department of Clinical Sciences and Translational Medicine, University of Rome Tor Vergata, \\ 00133 Rome, Italy; valentina.delprete90@libero.it (V.D.P.); david.dicave@unicamillus.org (D.D.C.) \\ 2 Department of Biomedicine and Prevention, University of Rome Tor Vergata, 00133 Rome, Italy; \\ lucaguarnera@live.com (L.G.); raffaele.f.palmieri@gmail.com (R.P.); luca.maurillo@uniroma2.it (L.M.); \\ francesco.buccisano@uniroma2.it (F.B.); adriano.venditti@uniroma2.it (A.V.); dlpmlr00@uniroma2.it (M.I.D.P.) \\ * Correspondence: giovangiacinto.paterno@alumni.uniroma2.eu; Tel.: +39-0620908215 or +39-0620908122 \\ + Presented at the XXXI National Conference of the Italian Society of Parasitology (SoIPa), Online, \\ 16-19 June 2021.
}

check for updates

Citation: Prete, V.D.; Paterno, G.; Di Cave, D.; Guarnera, L.; Palmieri, R.; Maurillo, L.; Buccisano, F.; Venditti, A.; Del Principe, M.I. Prevalence and Genotyping of Pneumocystis jirovecii Pneumonia in Patients with Previously Untreated Acute Myeloid Leukemia. Biol. Life Sci. Forum 2021, 5 , 3. https://doi.org/10.3390/ blsf2021005003

Academic Editors: Donato Traversa, Angela Di Cesare and Simone Morelli

Published: 20 October 2021

Publisher's Note: MDPI stays neutral with regard to jurisdictional claims in published maps and institutional affiliations.

Copyright: (c) 2021 by the authors. Licensee MDPI, Basel, Switzerland. This article is an open access article distributed under the terms and conditions of the Creative Commons Attribution (CC BY) license (https:// creativecommons.org/licenses/by/ $4.0 /)$.

\begin{abstract}
Pneumocystis jirovecii pneumonia (PJP) is an opportunistic infection affecting immunocompromised patients. Patients with acute myeloid leukemia (AML) are not considered at high risk of PJP, thus, prophylaxis is not recommended. Between 2010 and 2020 we retrospectively analyzed 251 AML patients. We performed molecular diagnosis and genotyping of Pneumocystis jirovecii in 67 bronchoalveolar lavage samples. Eleven cases of PJP were diagnosed, with a prevalence of $4.3 \%$. Our study confirms that the most widespread genotype in Europe is genotype 1; in our patients, $70 \%$ presented with genotype 1 and $30 \%$ the genotype 3 .
\end{abstract}

Keywords: Pnemocystis jirovecii pneumonia; acute myeloid leukemia; bronchoalveolar lavage; genotyping

\section{Introduction}

Pneumocystis jirovecii (PJ) is an ascomycetous fungus that causes opportunistic infections in humans. PJ pneumonia (PJP) is a life-threatening interstitial pneumonia that mostly occurs in immunocompromised patients such as those with acquired immunodeficiency syndrome (AIDS) and lymphoproliferative disorders, as well as allogeneic hematopoietic stem cell transplantation (HSCT) recipients [1-3]. Since 2005, the European Conference on Infections in Leukemia (ECIL) has aimed to produce recommendations for the management of infections in patients with hematologic malignancies (HM) [4]. The ECIL recommend prophylaxis with daily or three-times weekly trimethoprim/sulfamethoxazole (TMP-SMX) in patients with strong evidence of high risk of PJP. The current guidelines do not extend to acute myeloid leukemia (AML); although PJP has been observed in patients with AML not receiving HSCT, data on incidence are sparce, and mostly based on case reports [5]. Generally, those patients are not considered at high risk of PJP development, due to the unusual administration of high dose of steroids during induction therapy [6]. This retrospective study aimed to evaluate the incidence of PJP during AML-induction therapy, and to evaluate the need for prophylaxis. Additionally, we performed molecular diagnosis and genotyping of PJ in bronchoalveolar lavage (BAL) samples [7].

\section{Materials and Methods}

We retrospectively analyzed 251 consecutive newly diagnosed adult ( $\geq 18$ years old) patients with non-promyelocytic AML, admitted between January 2010 and May 2020 to 
the Hematology Ward of the "Policlinico Universitario di Roma Tor Vergata" to receive induction therapy. High-resolution computed tomography (HRCT) of the chest was routinely performed in all patients as part of their initial evaluation before the start of AML-induction therapy, in cases of fever persisting for more than 72 hours following antibiotic therapy initiation or with the presence of respiratory signs/chest pains not otherwise explained [8]. Sixty-seven BALs were performed in patients with pneumonia of unclear etiology or nonresponse to antibiotics [9]. BAL samples were evaluated at the parasitology laboratory, using the mtLSU-rRNA gene as a diagnostic marker for the detection of $P$. jirovecii and the determination of the specific genotype of the species [10]. Molecular analysis was carried out by PCR amplification and subsequent sequencing to highlight single nucleotide polymorphisms (SNP) in the sequence. Amplified DNA was sequenced by Bio-Fab Research and multiple alignments were performed using Clustal W2 and MEGA7 software to find a correlation between the genotype and the patient's characteristics. The mtLSU-rRNA locus distinguished 4 genotypes on the basis of polymorphisms in positions 85 and 248: genotype $1(85: C / 248: C)$, genotype $2(85: A / 248: C)$, genotype $3(85: T / 248: C)$ and genotype 4 (85:C/248:T) [11]. All patients have consented, through informed consent, to the processing of their sensitive data.

\section{Results}

During the study period, 251 new diagnoses of non-promyelocytic AML were made (153 males and 98 females, median age 62); of these, 172 were submitted to intensive chemotherapy, 43 to hypomethylating agents or low dose cytarabine, and 36 were found unfit to receive active AML treatment and received only supportive therapy. None of the patients received steroids for more than 15 days, and the maximum dosage used was $20 \mathrm{mg}$ prednisone/day. None of the patients had received previous TMP/SMX prophylaxis. Sixty-seven BALs were performed (Table 1). PJ presence was demonstrated in 11/67 (16.7\%) of BALs with a prevalence of $4.3 \%(11 / 251,11$ male), median age 71 (range 63-84 years). In our patients, $8 / 11(73 \%)$ presented with genotype 1 and $3 / 11(27 \%)$ with genotype 3 . No statistically significant correlations have yet been found between these patients.

Table 1. Patient characteristics.

\begin{tabular}{cc}
\hline N. of Patients & 251 \\
Sex & \\
Male & $153(61 \%)$ \\
Female & $98(39 \%)$ \\
\hline Age & Median: 62 yrs \\
$>60$ yrs & $145(57.8 \%)$ \\
$>60$ yrs & $106(42.2 \%)$ \\
\hline BAL performed: & 67 \\
Prior to first-line therapy & $39(58.2 \%)$ \\
After first-line therapy & $28(43.3 \%)$ \\
\hline AML Treatment & $179(71.3 \%)$ \\
Intensive chemotherapy & $36(14.3 \%)$ \\
Non-intensive chemotherapy & $36(14.3 \%)$ \\
Supportive care & $11 / 67(16.4 \%)$ \\
\hline PJ + BAL & $2 / 39(5.1 \%)$ \\
Prior to first-line therapy & $9 / 28(32 \%)$ \\
After first-line therapy & $105(41.8 \%)$ \\
\hline Comorbidities & $146(58.2 \%)$ \\
\hline None &
\end{tabular}


Table 1. Cont.

\begin{tabular}{cc}
\hline N. of Patients & $\mathbf{2 5 1}$ \\
\hline Co-present lung infections & \\
Aspergillosis & $5 / 11(45.5 \%)$ \\
Bacterial pneumonia & $1 / 11(9 \%)$ \\
CMV & $1 / 11(9 \%)$ \\
None & $3 / 11(27.3 \%)$ \\
\hline Lung HRTC scan findings \\
Typical \\
Atypical & $6 / 11(54.5 \%)$ \\
BAL, bronchoalveolar lavage; CMV, cytomegalovirus; HRTC, High-resolution computed tomography; PJ, \\
Pneumocystis jirovecii.
\end{tabular}

\section{Conclusions}

PJP is an opportunistic fungal infection commonly found in HM with wide variability based on the type of malignancy and treatment. In our experience, the incidence of PJP during AML induction therapy was not negligible (4.3\% among the whole series of patients and $16.7 \%$ of pneumonias of unknown origin or unresponsive to antibiotics). Accordingly, our study showed that surveillance is important and awareness of PJP in AML patients is necessary. Prophylaxis for PJP, which results in mortality benefits when the estimated risk of infection exceeds 3.5\%, as previously reported, should be considered [12]. From a molecular point of view, our study confirms that the most widespread genotype in Europe is genotype 1 [10]. Further prospective studies are needed to assess the actual indications for prophylaxis, to evaluate PJP incidence during the subsequent course of AML and to assess the exact timing for prophylaxis initiation and duration.

Author Contributions: All authors contributed equally. All authors have read and agreed to the published version of the manuscript.

Funding: This research received no external funding.

Informed Consent Statement: Informed consent was obtained from all subjects involved in the study.

Conflicts of Interest: The authors declare no conflict of interest.

\section{References}

1. Catherinot, E.; Lanternier, F.; Bougnoux, M.-E.; Lecuit, M.; Couderc, L.-J.; Lortholary, O. Pneumocystis jirovecii Pneumonia. Infect. Dis. Clin. N. Am. 2010, 24, 107-138. [CrossRef] [PubMed]

2. $\quad$ Evernden, C.; Dowhan, M.; Dabas, R.; Chaudhry, A.; Kalra, A.; Dharmani-Khan, P.; Gregson, D.; Johnson, A.; Jupp, J.; JimenezZepeda, V.; et al. High incidence of Pneumocystis jirovecii pneumonia in allogeneic hematopoietic cell transplant recipients in the modern era. Cytotherapy 2020, 22, 27-34. [CrossRef] [PubMed]

3. Roux, A.; Canet, E.; Valade, S.; Gangneux-Robert, F.; Hamane, S.; Lafabrie, A.; Maubon, D.; Debourgogne, A.; Le Gal, S.; Dalle, F.; et al. Pneumocystis jiroveciiPneumonia in Patients with or without AIDS, France. Emerg. Infect. Dis. 2014, 20, 1490-1497. [CrossRef] [PubMed]

4. Alanio, A.; Hauser, P.; Lagrou, K.; Melchers, W.J.G.; Helweg-Larsen, J.; Matos, O.; Cesaro, S.; Maschmeyer, G.; Einsele, H.; Donnelly, J.P.; et al. ECIL guidelines for the diagnosis of Pneumocystis jirovecii pneumonia in patients with haematological malignancies and stem cell transplant recipients. J. Antimicrob. Chemother. 2016, 71, 2386-2396. [CrossRef] [PubMed]

5. Szer, J. The Journal in 2017. Intern. Med. J. 2018, 48, 7-8. [CrossRef] [PubMed]

6. Pagano, L.; Fianchi, L.; Mele, L.; Girmenia, C.; Offidani, M.; Ricci, P.; Mitra, M.E.; Picardi, M.; Caramatti, C.; Piccaluga, P.; et al. Pneumocystis carinii pneumonia in patients with malignant haematological diseases: 10 years' experience of infection in GIMEMA centres. Br. J. Haematol. 2002, 117, 379-386. [CrossRef] [PubMed]

7. Fréalle, E.; Valade, S.; Guigue, N.; Hamane, S.; Chabé, M.; Le Gal, S.; Damiani, C.; Totet, A.; Aliouat, E.M.; Nevez, G.; et al. Diffusion of Pneumocystis jirovecii in the surrounding air of patients with Pneumocystis colonization: frequency and putative risk factors: Table 1. Med. Mycol. 2016, 55, 568-572. [CrossRef] [PubMed]

8. Kanne, J.P.; Yandow, D.R.; Meyer, C.A. Pneumocystis jiroveci Pneumonia: High-Resolution CT Findings in Patients with and Without HIV Infection. Am. J. Roentgenol. 2012, 198, W555-W561. [CrossRef] [PubMed] 
9. Marchesi, F.; Cattaneo, C.; Criscuolo, M.; Delia, M.; Dargenio, M.; Del Principe, M.I.; Spadea, A.; Fracchiolla, N.S.; Melillo, L.; Perruccio, K.; et al. A bronchoalveolar lavage-driven antimicrobial treatment improves survival in hematologic malignancy patients with detected lung infiltrates: A prospective multicenter study of the SEIFEM group. Am. J. Hematol. 2019, 94, 1104-1112. [CrossRef] [PubMed]

10. Alanio, A.; Gits-Muselli, M.; Guigue, N.; Desnos-Ollivier, M.; Calderon, E.J.; Di Cave, D.; Dupont, D.; Hamprecht, A.; Hauser, P.M.; Helweg-Larsen, J.; et al. Diversity of Pneumocystis jirovecii Across Europe: A Multicentre Observational Study. EBioMedicine 2017, 22, 155-163. [CrossRef] [PubMed]

11. Dimonte, S.; Berrilli, F.; D’Orazi, C.; D'Alfonso, R.; Placco, F.; Bordi, E.; Perno, C.F.; Di Cave, D. Molecular analysis based on mtLSU-rRNA and DHPS sequences of Pneumocystis jirovecii from immunocompromised and immunocompetent patients in Italy. Infect. Genet. Evol. 2013, 14, 68-72. [CrossRef] [PubMed]

12. Green, H.; Paul, M.; Vidal, L.; Leibovici, L. Prophylaxis of Pneumocystis Pneumonia in Immunocompromised Non-HIV-Infected Patients: Systematic Review and Meta-analysis of Randomized Controlled Trials. Mayo Clin. Proc. 2007, 82, 1052-1059. [CrossRef] [PubMed] 